\title{
Constraining dimension-six nonminimal Lorentz-violating electron-nucleon interactions with EDM physics
}

\author{
Jonas B. Araujo, ${ }^{1,2, *}$ A. H. Blin, ${ }^{3, \dagger}$ Marcos Sampaio, ${ }^{2,4, \$}$ and Manoel M. Ferreira, Jr. ${ }^{1, \S}$ \\ ${ }^{1}$ Departamento de Física, Universidade Federal do Maranhão, Campus Universitário do Bacanga, \\ São Luís-MA, 65080-805, Brazil \\ ${ }^{2}$ Centre for Particle Theory, University of Durham, Durham, DH1 3LE, United Kingdom \\ ${ }^{3}$ CFisUC, Department of Physics, University of Coimbra, P-3004-516 Coimbra, Portugal \\ ${ }^{4} \mathrm{CCNH}$, Universidade Federal do ABC, 09210-580, Santo André-SP, Brazil
}

(Received 28 February 2019; published 29 July 2019)

\begin{abstract}
The electric dipole moment (EDM) of an atom could arise also from $P$-odd and $T$-odd electron-nucleon couplings. In this work we investigate a general class of dimension-6 electron-nucleon $(e-N)$ nonminimal interactions mediated by Lorentz-violating (LV) tensors of rank ranging from 1 to 4 . The possible couplings are listed as well as their behavior under $C, P$, and $T$, allowing us to select the couplings compatible with EDM physics. The unsuppressed contributions of these couplings to the atom's Hamiltonian can be read as EDM-equivalent. The LV coefficients' magnitudes are limited using EDM experimental data to the level of $3.2 \times 10^{-13}(\mathrm{GeV})^{-2}$ or $1.6 \times 10^{-15}(\mathrm{GeV})^{-2}$.
\end{abstract}

DOI: 10.1103/PhysRevD.100.015046

\section{INTRODUCTION}

Electric dipole moments (EDMs) are excellent probes for violation of discrete symmetries [1-4] and of physics beyond the standard model (SM) [5,6]. EDM terms violate both parity $(P)$ and time reversal $(T)$ symmetries, while preserve charge conjugation $(C)$, if the $C P T$ theorem holds. In the SM structure, $P$ and $C P$ violations have been proposed and detected since the 1950s, but violations of $T$ only recently have been measured by the $B A B A R$ Collaboration [7]. Furthermore, the role of $C P$ violation is crucial in explaining the baryon asymmetry in the universe, as pointed out by Sakharov [8]. However, as a standard EDM is $C$-even, it cannot play a role by itself on the baryon asymmetry in the universe. Concerning the EDM experiments, the growing experimental precision has lead to stringent upper bounds on several $C P$-violating theories [9-12]. If an atomic EDM were to be detected, it could arise from intrinsic properties of the electrons and/or nucleus, or from $P$-odd and $T$-odd electron-nucleon $(e-N)$ couplings.

\footnotetext{
*jonas.araujo88@gmail.com

alex@uc.pt

marcos.sampaio@ufabc.edu.br

${ }^{\S}$ manojr.ufma@gmail.com
}

Published by the American Physical Society under the terms of the Creative Commons Attribution 4.0 International license. Further distribution of this work must maintain attribution to the author(s) and the published article's title, journal citation, and DOI. Funded by SCOAP ${ }^{3}$.
In the SM, the electronic EDM is generated via radiative corrections at four loop order [13,14], with magnitude $d_{e-\mathrm{SM}} \simeq 10^{-38} e \cdot \mathrm{cm}$. In addition, the current experimental measurement has ruled out an electronic EDM up to $d_{e-\exp }=1.1 \times 10^{-31} e \cdot \mathrm{cm}[9]$, which is still 7 orders of magnitude above the SM prediction. Concerning the EDM searches, it is important to remember that the carrier of EDM may be the electron or the atomic nucleus. In fact, the atomic nucleus, due to $P$-odd and $T$-odd nuclear interactions [15], can yield a nuclear EDM, which, for a pointlike nucleus, would be screened by the very interesting Schiff's theorem [16]. For a finite-sized nucleus, however, the screening is not total, and the Schiff moment appears as a residual effect [15]. Furthermore, if relativity is considered, not only the screening is ineffective, but the whole atom's or molecule's EDM may exceed the electron's by a few orders of magnitude [17-20]. Recent experiments to measure nuclear EDM have been undertaken and an upper bound of $d_{199} \mathrm{Hg}<7.4 \times 10^{-30} e \cdot \mathrm{cm}$ was set for the ${ }^{199} \mathrm{Hg}$ nucleus [21]. The surprisingly minute nuclear EDM, which is related to the QCD $\theta$-term, poses the strong $C P$ problem, whose solution could involve the yet undetected axions [22].

There are three ways to generate EDM for an atom. Two of them rely on EDM sources in the atom's constituents and appropriate means of circumventing the Schiff's theorem: (i) relativistic enhancement of the electron EDM; (ii) partial screening of the nuclear EDM due to the finite-sized nucleus. The third mechanism is based on $P$-odd and $T$-odd electron-nucleon interactions. Thus, even 
in an atom deprived of particle EDM sources-(i) and (ii), an effective atomic EDM may be engendered via dimension-6 interactions between the nucleus and the electronic cloud, being represented by $P$-odd and $T$-odd electron-nucleon $(e-N)$ couplings, such as

$$
\begin{aligned}
\mathcal{L}_{C P}= & i \frac{G_{F}}{\sqrt{2}} \sum_{j}\left[C_{S} \bar{N}_{j} N_{j} \bar{\psi} \gamma^{5} \psi+C_{P} \bar{N}_{j} \gamma^{5} N_{j} \bar{\psi} \psi\right. \\
& \left.+C_{T} \bar{N}_{j} \sigma^{\mu \nu} N_{j} \bar{\psi} \sigma^{\mu \nu} \gamma^{5} \psi\right] .
\end{aligned}
$$

Among these, the dominant contribution arises from the scalar-pseudoscalar coupling, $C_{S} \bar{N}_{j} N_{j} \bar{\psi} \gamma^{5} \psi$, where $G_{F}$ denotes Fermi's constant $[4,14,23,24]$. By supposing that the $e-N$ couplings are the sole source of EDM for the atom, the dimensionless coefficient $C_{S}$ can be constrained to the level $C_{S}<7.3 \times 10^{-10}$, according to the most precise experiment up to date on the electron's EDM [9]. Moreover, the scalar-pseudoscalar coupling is similar to the dominant term in ordinary atomic parity nonconservation (PNC), originated from the coupling of the axial electronic neutral weak current to the vector nucleonic neutral weak current via a $Z^{0}$ exchange $[4,24,25]$. The effects on atomic polarization in heavy atoms have also been studied [2,26,27]. $P$-odd and $T$-odd interactions in atomic systems may yield non-null matrix elements in heavy atoms with one valence electron [28]. Other $e-N$ couplings, including the tensor-pseudotensor and pseudoscalar-scalar, are investigated via atomic calculations for the ${ }^{199} \mathrm{Hg}$ nucleus [29].

EDM phenomenology can also arise in a Lorentzviolating (LV) scenario, addressed within the framework of the Standard Model extension (SME), developed by Kostelecky, Colladay and others in Refs. [30]. The SME includes dimension-4 and dimension-3 LV terms in all sectors of the SM, comprising fermions [31-34], photons [35-37], Yang-Mills developments [38], Casimir effect [39], photon-fermion interactions [40,41] and electroweak (EW) processes [42-44]. The minimal SME can be further extended so as to contain nonminimal couplings composed of higher-order derivatives in the photon [45] and in the fermion sector [46], as well as higher-dimension operators [47-49]. Nonminimal couplings deprived of higher-order derivatives (except the one contained in the field strength) have been proposed in describing LV interactions between fermions and photons [50-52] and LV interactions in the electroweak sector [53]. Dimension-5 terms of MyersPospelov type have also been investigated in the context of black-body radiation [54] and emission of electromagnetic and gravitational waves [55].

Lorentz violation can also work as a source of $C P$ violation and EDM via radiative corrections [56] or even at tree level via dimension-5 nonminimal couplings [57-60]. Lorentz-violating (LV) dimension-5 nonminimal couplings have been proposed as nonusual QED interactions between fermions and photons, yielding EDM Lagrangians pieces as $\lambda \bar{\psi}\left(K_{F}\right)_{\mu \nu \alpha \beta} \Gamma^{\mu \nu} F^{\alpha \beta} \psi$ and $\lambda_{1} \bar{\psi} T_{\mu \alpha} F^{\alpha}{ }_{\nu} \Gamma^{\mu \nu} \psi$, where $\left(K_{F}\right)_{\mu \nu \alpha \beta}$ and $T_{\mu \alpha}$ are $C P T$-even LV tensors, and $\Gamma^{\mu \nu}$ are combinations of Dirac matrices [58-60]. Electron EDM experimental data has yielded upper bounds as tight as $10^{-25}(\mathrm{eV})^{-1}$ on the magnitude of these couplings. Looking at another route, nuclear EDMs may also be connected with LV physics. Lorentz-violating contributions to the nuclear Schiff moment have been investigated as well [61]. It is worth mentioning that LV theories were also analyzed in connection with the magnetic dipole moment physics [6264], notwithstanding providing less severe upper bounds.

In this work, we investigate a class of dimension- 6 and Lorentz-violating $e-N$ couplings, composed by rank-1, rank-2, rank-3, and rank-4 background tensors, and the possible generation of atomic EDM. These couplings were first proposed in a recent generalization of gauge theories with LV operators of arbitrary dimension [65], which contains nonminimal couplings of dimensions ranging from 5 to 8 . Specifically, we are interested in the dimension-6 fermion-fermion interactions displayed here in Tables I and IV, for the purpose of generating EDM. This work is organized as follows. In Sec. II, several possibilities of dimension- 6 couplings are presented and analyzed concerning their behavior under $C, P$, and $T$ operations and suppression criteria for yielding EDM. Also, redundancies in the couplings are illustrated and commented. In Sec. III, we examine the Hamiltonians corresponding to unsuppressed EDM couplings, and their respective energy shifts are estimated and limited using the current experimental data. The sidereal analysis on the LV terms is also performed. In Sec. IV, the conclusions are drawn.

\section{NONMINIMAL ELECTRON-NUCLEON LORENTZ-VIOLATING COUPLINGS}

An atomic EDM could be the result of the EDM contained in the electrons or nucleons, or it could be

TABLE I. General CPT-odd couplings with a rank-1 LV tensor and Dirac bilinears. NRL stands for the nonrelativistic limit for the nucleons $(N)$. In this limit, the coupling component can be suppressed, "S," or not suppressed, "NS."

\begin{tabular}{lccc}
\hline \hline Coupling & $P$-odd, $T$-odd piece & NRL & EDM \\
\hline$\left(k_{S V}\right)_{\mu}(\bar{N} N)\left(\bar{\psi} \gamma^{\mu} \psi\right)$ & $\left(k_{S V}\right)_{i}(\bar{N} N)\left(\bar{\psi} \gamma^{i} \psi\right)$ & NS & Yes \\
$\left(k_{V S}\right)_{\mu}\left(\bar{N} \gamma^{\mu} N\right)(\bar{\psi} \psi)$ & $\left(k_{V S}\right)_{i}\left(\bar{N} \gamma^{i} N\right)(\bar{\psi} \psi)$ & $\mathrm{S}$ & $\ldots$ \\
$\left(k_{V P}\right)_{\mu}\left(\bar{N} \gamma^{\mu} N\right)\left(\bar{\psi} i \gamma_{5} \psi\right)$ & $\left(k_{V P}\right)_{0}\left(\bar{N} \gamma^{0} N\right)\left(\bar{\psi} i \gamma_{5} \psi\right)$ & NS & Yes \\
$\left(k_{P V}\right)_{\mu}\left(\bar{N} i \gamma_{5} N\right)\left(\bar{\psi} \gamma^{\mu} \psi\right)$ & $\left(k_{P V}\right)_{0}\left(\bar{N} \gamma_{5} N\right)\left(\bar{\psi} \gamma^{0} \psi\right)$ & $\mathrm{S}$ & $\ldots$ \\
$\left(k_{S A}\right)_{\mu}(\bar{N} N)\left(\bar{\psi} \gamma^{\mu} \gamma_{5} \psi\right)$ & None & $\ldots$ & $\ldots$ \\
$\left(k_{A S}\right)_{\mu}\left(\bar{N} \gamma^{\mu} \gamma_{5} N\right)(\bar{\psi} \psi)$ & None & $\ldots$ & $\ldots$ \\
$\left(k_{P A}\right)_{\mu}\left(\bar{N} i \gamma_{5} N\right)\left(\bar{\psi} \gamma^{\mu} \gamma_{5} \psi\right)$ & None & $\ldots$ & $\ldots$ \\
$\left(k_{A P}\right)_{\mu}\left(\bar{N} \gamma^{\mu} \gamma_{5} N\right)\left(\bar{\psi} i \gamma_{5} \psi\right)$ & None & $\ldots$ & $\ldots$ \\
\hline \hline
\end{tabular}


due to $P$-odd and $T$-odd electron-nucleon interactions only. Lorentz violation is a natural source of $C P$-breaking and can work as an environment to generate $P$-odd and $T$-odd $e-N$ interactions. In this sense, we are interested in a class of Lorentz-violating (LV) electron-nucleon couplings. Considering dimension- 6 couplings involving 2 fermions, we are restricted to derivative free couplings, otherwise these would have dimension higher than 6 , one unit higher for each extra derivative considered. The simplest case involves a rank-1 LV tensor $\left(k_{X X}\right)_{\mu}$, so that the effective dimension-6 Lagrangian piece should have the form

$$
\mathcal{L}_{\mathrm{LV}}=\left(k_{X X}\right)_{\mu}\left[\left(\bar{N} \Gamma_{1} N\right)\left(\bar{\psi} \Gamma_{2} \psi\right)\right]^{\mu},
$$

indicating that the upper index $\mu$ belongs to either $\Gamma_{1}$ or $\Gamma_{2}$. In addition, the subscript $X X$ in $\left(k_{X X}\right)_{\mu}$ refers to the nature of the two fermion bilinears as follows: scalar $(S)$, pseudoscalar $(P)$, vector $(V)$, axial vector $(A)$, and tensor $(T)$, which account for the 16 linearly independent $4 \times 4$ matrices. Due to the limitation on dimension- 6 couplings, the operators $\Gamma_{1,2}$ must be combinations of Dirac matrices. We use the definitions

$$
\begin{array}{ll}
\alpha^{i}=\left(\begin{array}{cc}
0 & \sigma^{i} \\
\sigma^{i} & 0
\end{array}\right), & \Sigma^{k}=\left(\begin{array}{cc}
\sigma^{k} & 0 \\
0 & \sigma^{k}
\end{array}\right), \\
\gamma^{0}=\left(\begin{array}{cc}
1 & 0 \\
0 & -1
\end{array}\right), & \gamma^{5}=\left(\begin{array}{cc}
0 & 1 \\
1 & 0
\end{array}\right),
\end{array}
$$

and $\sigma^{\mu \nu}=i\left[\gamma^{\mu}, \gamma^{\nu}\right] / 2$, with $\sigma^{0 j}=i \alpha^{j}, \sigma^{i j}=\epsilon_{i j k} \Sigma^{k}$. As we are interested in the generation of EDM, we should focus on the $P$-odd and $T$-odd couplings. In principle, one can have $\Gamma_{1}, \Gamma_{2}=\gamma^{\mu}, \gamma^{\mu} \gamma^{5}, \gamma^{5}, 1$, which provide several possibilities,

$$
\begin{aligned}
& \Gamma_{1}=\gamma^{\mu}, \quad \Gamma_{2}=1 ; \quad \Gamma_{1}=\gamma^{\mu}, \quad \Gamma_{2}=\gamma^{5} ; \\
& \Gamma_{1}=\gamma^{\mu} \gamma^{5}, \quad \Gamma_{2}=1 ; \quad \Gamma_{1}=\gamma^{\mu} \gamma^{5}, \quad \Gamma_{2}=\gamma^{5} ;
\end{aligned}
$$

and the corresponding combinations interchanging $\Gamma_{1}$ and $\Gamma_{2}$, yielding eight couplings that are listed in Table I.

$C P T$-odd dimension- 6 couplings, containing the tensor operator $\sigma^{\mu \nu}$, can also be proposed. The initial options would be

$$
\Gamma_{1}=\gamma^{\mu}, \quad \Gamma_{2}=\sigma^{\mu \nu} ; \quad \Gamma_{1}=\gamma^{\mu} \gamma^{5}, \quad \Gamma_{2}=\sigma^{\mu \nu}
$$

to which one adds the corresponding combinations letting $\Gamma_{1} \leftrightarrow \Gamma_{2}$, engendering four possibilities, displayed in Table II. These couplings, however, are included in a rank-3 generalization. Observe, for instance:

$$
\begin{aligned}
\left(\kappa_{V T}\right)_{\nu}\left(\bar{N} \gamma_{\mu} N\right)\left(\bar{\psi} \sigma^{\mu \nu} \psi\right) & =\left(\kappa_{V T}\right)_{\nu} g_{\beta \mu}\left(\bar{N} \gamma^{\mu} N\right)\left(\bar{\psi} \sigma^{\beta \nu} \psi\right) \\
& =\left(k_{V T}\right)_{\nu \beta \mu}\left(\bar{N} \gamma^{\mu} N\right)\left(\bar{\psi} \sigma^{\beta \nu} \psi\right),
\end{aligned}
$$

TABLE II. Redundant $C P T$-odd couplings with a rank-1 LV tensor and matrixes $\gamma^{\mu}, \sigma^{\mu \nu}$, and $\gamma^{5}$.

\begin{tabular}{lccc}
\hline \hline Coupling & $P$-odd, $T$-odd piece & NRL & EDM \\
\hline$\left(k_{V T}\right)_{\nu}\left(\bar{N} \gamma_{\mu} N\right)\left(\bar{\psi} \sigma^{\mu \nu} \psi\right)$ & None & $\ldots$ & $\ldots$ \\
$\left(k_{A T}\right)_{\nu}\left(\bar{N} \gamma_{\mu} \gamma_{5} N\right)\left(\bar{\psi} \sigma^{\mu \nu} \psi\right)$ & $\left(k_{A T}\right)_{0}\left(\bar{N} \gamma_{i} \gamma_{5} N\right)\left(\bar{\psi} \sigma^{i 0} \psi\right)$ & NS & Yes \\
$\left(k_{T V}\right)_{\nu}\left(\bar{N} \sigma^{\mu \nu} N\right)\left(\bar{\psi} \gamma_{\mu} \psi\right)$ & None & $\ldots$ & $\ldots$ \\
$\left(k_{T A}\right)_{\nu}\left(\bar{N} \sigma^{\mu \nu} N\right)\left(\bar{\psi} \gamma_{\mu} \gamma_{5} \psi\right)\left(k_{T A}\right)_{0}\left(\bar{N} \sigma^{i 0} N\right)\left(\bar{\psi} \gamma_{i} \gamma_{5} \psi\right)$ & $\mathrm{S}$ & $\cdots$ \\
\hline \hline
\end{tabular}

where $\left(k_{V T}\right)_{\nu \beta \mu}=\left(k_{V T}\right)_{\nu} g_{\beta \mu}$ is a particular parametrization, with $g_{\beta \mu}$ being the Minkowski metric tensor. Consequently, all the rank-1 couplings with one contracted index between the bilinears can be reproduced by the rank-3 structures of Table IV. In other words, all the rank-1 couplings of Table II are included as particular cases of the rank-3 couplings to be shown in Table IV, which also includes the couplings of rank-2 and rank-4.

Other combinations involving $\sigma^{\mu \nu}$, such as

$$
\begin{array}{cc}
\Gamma_{1}=\gamma_{\nu} \sigma^{\mu \nu}, & \Gamma_{2}=1, \\
\Gamma_{1}=\gamma_{\nu} \sigma^{\mu \nu}, & \Gamma_{2}=\gamma^{5}, \\
\Gamma_{1}=\gamma_{\nu} \sigma^{\mu \nu} \gamma^{5}, & \Gamma_{2}=1,
\end{array}
$$

could also be proposed, but dot not bring novelty, due to the redundancy of the product $\gamma_{\nu} \sigma^{\mu \nu}=3 i \gamma^{\mu}$. In addition, the identity

$$
\sigma_{\mu \nu} \gamma_{5}=\frac{i}{2} \epsilon_{\mu \nu \rho \theta} \sigma^{\rho \theta}
$$

where $\epsilon_{\mu \nu \rho \theta}$ (with $\epsilon_{0123}=+1$ ) is the Levi-Civita symbol, frees us from taking "pseudotensor" $\left(\sigma_{\mu \nu} \gamma_{5}\right)$ terms into account, since these can be written in terms of the tensor ones, as follows:

$$
\left(\left(k_{V-P T}\right)^{\nu}\right)\left(\bar{N} \gamma^{\mu} N\right)\left(\bar{\psi} i \sigma_{\mu \nu} \gamma_{5} \psi\right)=\left(k_{V T}\right)_{\mu \rho \theta}\left(\bar{N} \gamma^{\mu} N\right)\left(\bar{\psi} \sigma^{\rho \theta} \psi\right),
$$

where

$$
\left(k_{V T}\right)_{\mu \rho \theta}=\frac{1}{2} \epsilon_{\nu \mu \rho \theta}\left(k_{V-P T}\right)^{\nu} .
$$

Thus, these couplings are equivalent to the rank- 3 ones displayed in Table V.

Concerning the couplings in Tables I and IV, these are considered suppressed when the nucleon's bilinear mixes large and small spinor components, becoming negligible in the nonrelativistic limit. We obviously are interested only in the unsuppressed, i.e., the bilinears composed of only large components. The behavior of the couplings under discrete symmetries depends on the way the Dirac bilinears 
TABLE III. Behavior of Dirac bilinears under discrete symmetry operators.

\begin{tabular}{ccccccccc}
\hline \hline & $\bar{\psi} \psi$ & $\bar{\psi} i \gamma_{5} \psi$ & $\bar{\psi} \gamma^{0} \psi$ & $\bar{\psi} \gamma^{i} \psi$ & $\bar{\psi} \gamma^{0} \gamma_{5} \psi$ & $\bar{\psi} \gamma^{i} \gamma_{5} \psi$ & $\bar{\psi} \sigma^{0 i} \psi$ & $\bar{\psi} \sigma^{i j} \psi$ \\
\hline$P$ & + & - & + & - & - & + & - & + \\
$T$ & + & - & + & - & + & - & + & - \\
$C$ & + & + & - & - & + & + & - & - \\
\hline \hline
\end{tabular}

transform under these symmetry operations, as shown in Table III.

It is important to stress that only the pieces that are simultaneously $P$-odd and $T$-odd can generate EDM. As the Lorentz-violating couplings presented in Tables I are $C P T$-odd, the simultaneously $P$-odd and $T$-odd pieces turn out to be $C P$-even, but able to generate EDM. Effectively, we are interested only in the unsuppressed $P$-odd and $T$-odd couplings, that is

$$
\left(k_{S V}\right)_{i}(\bar{N} N)\left(\bar{\psi} \gamma \gamma^{i} \psi\right), \quad\left(k_{V P}\right)_{0}\left(\bar{N} \gamma^{0} N\right)\left(\bar{\psi} i \gamma_{5} \psi\right),
$$

where the factor of $i$ was inserted in order to guarantee the hermicity. The $C P T$-odd effective Lagrangian, for the possible couplings involving a rank-1 LV tensor, is then

$\mathcal{L}_{\mathrm{LV}-1}=\sum_{N}\left[\left(k_{S V}\right)_{i}(\bar{N} N)\left(\bar{\psi} \gamma^{i} \psi\right)+\left(k_{V P}\right)_{0}\left(\bar{N} \gamma^{0} N\right)\left(\bar{\psi} i \gamma_{5} \psi\right)\right]$

Following the rank-1 case, it is interesting to analyze now the $e-N$ couplings composed by a rank-2 LV tensor, $\left(k_{X X}\right)_{\mu \nu}$, which are, obviously, $C P T$-even. All possibilities are listed in Table IV, which contains two $P$-odd and $T$-odd $e-N$ couplings that are not suppressed in the nonrelativistic limit for the nucleons. These couplings are $C P$-odd, as the usual Lorentz-preserving EDM terms. Again, similarly as in Eq. (6), couplings with a rank-2 tensor presenting index contractions between the bilinears can be read as particular cases of the rank-4 generalization, presented in Table IV. That said, the $C P T$-even effective Lagrangian is

TABLE IV. General couplings with LV tensors of ranks 2, 3, and 4, and Dirac bilinears. Again, NRL stands for the nonrelativistic limit for the nucleons. Also, in this limit, "S" and "NS" stand for suppressed and not suppressed, respectively.

\begin{tabular}{|c|c|c|c|}
\hline Coupling & $P$-odd and $T$-odd piece & NRL & EDM \\
\hline \multicolumn{4}{|l|}{ Rank-2 } \\
\hline \multirow[t]{2}{*}{$\left(k_{V V}\right)_{\mu \nu}\left(\bar{N} \gamma^{\mu} N\right)\left(\bar{\psi} \gamma^{\nu} \psi\right)$} & $\left(k_{V V}\right)_{i 0}\left(\bar{N} \gamma^{i} N\right)\left(\bar{\psi} \gamma^{0} \psi\right)$ & $\mathrm{S}$ & $\cdots$ \\
\hline & $\left(k_{V V}\right)_{0 i}\left(\bar{N} \gamma^{0} N\right)\left(\bar{\psi} \gamma^{i} \psi\right)$ & NS & Yes \\
\hline$\left(k_{A V}\right)_{\mu \nu}\left(\bar{N} \gamma^{\mu} \gamma_{5} N\right)\left(\bar{\psi} \gamma^{\nu} \psi\right)$ & None & $\ldots$ & $\ldots$ \\
\hline$\left(k_{V A}\right)_{\mu \nu}\left(\bar{N} \gamma^{\mu} N\right)\left(\bar{\psi} \gamma^{\nu} \gamma_{5} \psi\right)$ & None & $\cdots$ & $\cdots$ \\
\hline \multirow[t]{2}{*}{$\left(k_{A A}\right)_{\mu \nu}\left(\bar{N} \gamma^{\mu} \gamma_{5} N\right)\left(\bar{\psi} \gamma \gamma^{\nu} \gamma_{5} \psi\right)$} & $\left(k_{A A}\right)_{0 i}\left(\bar{N} \gamma^{0} \gamma_{5} N\right)\left(\bar{\psi} \gamma^{i} \gamma_{5} \psi\right)$ & $\mathrm{S}$ & $\cdots$ \\
\hline & $\left(k_{A A}\right)_{i 0}\left(\bar{N} \gamma^{i} \gamma_{5} N\right)\left(\bar{\psi} \gamma^{0} \gamma_{5} \psi\right)$ & NS & Yes \\
\hline$\left(k_{T S}\right)_{\mu \nu}\left(\bar{N} \sigma^{\mu \nu} N\right)(\bar{\psi} \psi \psi)$ & None & $\cdots$ & $\cdots$ \\
\hline$\left(k_{T P}\right)_{\mu \nu}\left(\bar{N} \sigma^{\mu \nu} N\right)\left(\bar{\psi} i \gamma_{5} \psi\right)$ & None & $\cdots$ & $\cdots$ \\
\hline$\left(k_{S T}\right)_{\mu \nu}(\bar{N} N)\left(\bar{\psi} \sigma^{\mu \nu} \psi\right)$ & None & $\ldots$ & $\ldots$ \\
\hline$\left(k_{P T}\right)_{\mu \nu}\left(\bar{N} i \gamma_{5} N\right)\left(\bar{\psi} \sigma^{\mu \nu} \psi\right)$ & None & $\cdots$ & $\cdots$ \\
\hline \multicolumn{4}{|l|}{ Rank-3 } \\
\hline$\left(k_{V T}\right)_{\alpha \mu \nu}\left(\bar{N} \gamma^{\alpha} N\right)\left(\bar{\psi} \sigma^{\mu \nu} \psi\right)$ & None & $\cdots$ & $\cdots$ \\
\hline \multirow[t]{3}{*}{$\left(k_{A T}\right)_{\alpha \mu \nu}\left(\bar{N} \gamma^{\alpha} \gamma_{5} N\right)\left(\bar{\psi} \sigma^{\mu \nu} \psi\right)$} & $\left(k_{A T}\right)_{0 i j}\left(\bar{N} \gamma^{0} \gamma_{5} N\right)\left(\bar{\psi} \sigma^{i j} \psi\right)$ & $\mathrm{S}$ & $\cdots$ \\
\hline & $\left(k_{A T}\right)_{i 0 j}\left(\bar{N} \gamma^{i} \gamma_{5} N\right)\left(\bar{\psi} \sigma^{0 j} \psi\right)$ & NS & Yes \\
\hline & $\left(k_{A T}\right)_{i j 0}\left(\bar{N} \gamma^{i} \gamma_{5} N\right)\left(\bar{\psi} \sigma^{j 0} \psi\right)$ & NS & Yes \\
\hline$\left(k_{T V}\right)_{\alpha \mu \nu}\left(\bar{N} \sigma^{\mu \nu} N\right)\left(\bar{\psi} \gamma^{\alpha} \psi\right)$ & None & $\ldots$ & $\ldots$ \\
\hline \multirow{3}{*}{$\left(k_{T A}\right)_{\alpha \mu \nu}\left(\bar{N} \sigma^{\mu \nu} N\right)\left(\bar{\psi} \gamma^{\alpha} \gamma_{5} \psi\right)$} & $\left(k_{T A}\right)_{0 i j}\left(\bar{N} \sigma^{i j} N\right)\left(\bar{\psi} \gamma^{0} \gamma_{5} \psi\right)$ & NS & Yes \\
\hline & $\left(k_{T A}\right)_{i 0 j}\left(\bar{N} \sigma^{0 j} N\right)\left(\bar{\psi} \gamma^{i} \gamma_{5} \psi\right)$ & $\mathrm{S}$ & $\cdots$ \\
\hline & $\left(k_{T A}\right)_{i j 0}\left(\bar{N} \sigma^{j 0} N\right)\left(\bar{\psi} \gamma^{i} \gamma_{5} \psi\right)$ & $\mathrm{S}$ & $\cdots$ \\
\hline \multicolumn{4}{|l|}{ Rank-4 } \\
\hline \multirow{4}{*}{$\left(k_{T T}\right)_{\alpha \beta \mu \nu}\left(\bar{N} \sigma^{\alpha \beta} N\right)\left(\bar{\psi} \sigma^{\mu \nu} \psi\right)$} & $\left(k_{T T}\right)_{0 i j k}\left(\bar{N} \sigma^{0 i} N\right)\left(\bar{\psi} \sigma^{j k} \psi\right)$ & $\mathrm{S}$ & $\ldots$ \\
\hline & $\left(k_{T T}\right)_{i 0 j k}\left(\bar{N} \sigma^{i 0} N\right)\left(\bar{\psi} \sigma^{j k} \psi\right)$ & $\mathrm{S}$ & $\ldots$ \\
\hline & $\left(k_{T T}\right)_{i j 0 k}\left(\bar{N} \sigma^{i j} N\right)\left(\bar{\psi} \sigma^{0 k} \psi\right)$ & NS & Yes \\
\hline & $\left(k_{T T}\right)_{i j k 0}\left(\bar{N} \sigma^{i j} N\right)\left(\bar{\psi} \sigma^{k 0} \psi\right)$ & NS & Yes \\
\hline
\end{tabular}




$$
\begin{aligned}
\mathcal{L}_{\mathrm{LV}-2}= & \sum_{N}\left[\left(k_{V V}\right)_{0 i}\left(\bar{N} \gamma^{0} N\right)\left(\bar{\psi} \gamma^{i} \psi\right)\right. \\
& \left.+\left(k_{A A}\right)_{i 0}\left(\bar{N} \gamma^{i} \gamma_{5} N\right)\left(\bar{\psi} \gamma^{0} \gamma_{5} \psi\right)\right] .
\end{aligned}
$$

After applying the nonrelativistic limit for the nucleons, using the definitions (3), the nucleon bilinears yield

$$
\begin{aligned}
\bar{N} N, \bar{N} \gamma^{0} N & \rightarrow n(\boldsymbol{r}), \\
\bar{N} \gamma_{i} \gamma_{5} N, \bar{N} i \sigma^{0 i} \gamma_{5} N & \rightarrow-\left\langle\sigma^{i}\right\rangle_{N} \cdot n(\boldsymbol{r}), \\
\bar{N} \sigma^{i j} N & \rightarrow \epsilon_{i j k}\left\langle\sigma^{k}\right\rangle_{N} \cdot n(\boldsymbol{r}),
\end{aligned}
$$

where $n(r)$ is the nucleon density, being the same for protons and neutrons, while $\left\langle\sigma^{k}\right\rangle_{N}$ is the effective average spin state of the nucleon. Notice that, while the densities add coherently to $A \cdot n(r)$, with $A$ being the atomic mass, the spins do not, so that only the (unpaired) valence nucleon will count. We can read from the Lagrangians (13) and (14) the possible EDM contributions via atomic parity nonconservation methods, which will be illustrated in Sec. III. For now, we can rewrite the effective Lagrangians (13) and (14) for the valence electron as follows:

$$
\mathcal{L}_{\mathrm{LVe}-1}=\left[\left(k_{S V}\right)_{i} A\left(\bar{\psi} \gamma^{i} \psi\right)+\left(k_{V P}\right)_{0} A\left(\bar{\psi} i \gamma_{5} \psi\right)\right] n(\boldsymbol{r}),
$$

and

$$
\mathcal{L}_{\mathrm{LVe}-2}=\left[A\left(k_{V V}\right)_{0 i}\left(\bar{\psi} \gamma^{i} \psi\right)+\left(k_{A A}\right)_{i 0}\left\langle\sigma^{i}\right\rangle_{N}\left(\bar{\psi} \gamma^{0} \gamma_{5} \psi\right)\right] n(\boldsymbol{r}) .
$$

Beyond the couplings with rank-1 and rank-2 LV tensors, we can explore possible interactions involving rank-3 and rank-4 tensors, which are displayed in Table IV. The $P$ - and $T$-odd pieces are shown, with their suppressed or unsuppressed parts. The rank-3 couplings of Table IV are $C P T$-odd, so that the highlighted $P$-odd and $T$-odd pieces are $C P$-even.

Besides the structures presented in Table IV, we could also propose couplings of the form:

$$
\left(k_{S X}\right)_{\alpha \mu \nu}(\bar{N} N)\left(\bar{\psi} \gamma^{\alpha} \sigma^{\mu \nu} \psi\right)
$$

Nevertheless, using the identity,

$$
\gamma^{\alpha} \sigma^{\mu \nu}=-i\left(g^{\alpha \nu} \gamma^{\mu}-g^{\mu \alpha} \gamma^{\nu}\right)+\epsilon^{\beta \alpha \mu \nu} \gamma_{\beta} \gamma_{5},
$$

the coupling (18) is reduced to structures as

$$
\left(k_{S V}\right)_{\alpha \mu}{ }^{\mu}(\bar{N} N)\left(\bar{\psi} \gamma^{\alpha} \psi\right), \quad\left(k_{S A}\right)_{\alpha \mu}{ }^{\mu}(\bar{N} N)\left(\bar{\psi} \gamma^{\alpha} \gamma_{5} \psi\right),
$$

which are equivalent to rank-1 couplings contained in Table I. Hence, the coupling (18) and its variations,

$$
\begin{aligned}
& \left(k_{S X}\right)_{\alpha \mu \nu}(\bar{N} N)\left(\bar{\psi} \gamma^{\alpha} \sigma^{\mu \nu} \psi\right), \quad\left(k_{S X}\right)_{\alpha \mu \nu}(\bar{N} N)\left(\bar{\psi} \gamma^{\alpha} \sigma^{\mu \nu} \gamma_{5} \psi\right), \\
& \left(k_{S X}\right)_{\alpha \mu \nu}\left(\bar{N} \gamma_{5} N\right)\left(\bar{\psi} \gamma^{\alpha} \sigma^{\mu \nu} \psi\right), \quad\left(k_{S X}\right)_{\alpha \mu \nu}\left(\bar{N} \gamma_{5} N\right)\left(\bar{\psi} \gamma^{\alpha} \sigma^{\mu \nu} \gamma_{5} \psi\right),
\end{aligned}
$$

will not be considered.

The couplings of Table IV will also be examined concerning the associated Hamiltonian and spectrum in the next section. In order to accomplish this, as already done for the rank-1 and rank-2 cases, we write the LV Lagrangian consisting of rank-3 and rank-4 tensor couplings:

$$
\begin{aligned}
\mathcal{L}_{\mathrm{LV}-3}= & \sum_{N}\left[\left(k_{A T}\right)_{i 0 j}\left(\bar{N} \gamma^{i} \gamma_{5} N\right)\left(\bar{\psi} \sigma^{0 j} \psi\right)\right. \\
& +\left(k_{A T}\right)_{i j 0}\left(\bar{N} \gamma^{i} \gamma_{5} N\right)\left(\bar{\psi} \sigma^{j 0} \psi\right) \\
& \left.+\left(k_{T A}\right)_{0 i j}\left(\bar{N} \sigma^{i j} N\right)\left(\bar{\psi} \gamma^{0} \gamma_{5} \psi\right)\right], \\
\mathcal{L}_{\mathrm{LV}-4}= & \sum_{N}\left[\left(K_{T T}\right)_{i j 0 k}\left(\bar{N} \sigma^{i j} N\right)\left(\bar{\psi} \sigma^{0 k} \psi\right)\right. \\
& \left.+\left(K_{T T}\right)_{i j k 0}\left(\bar{N} \sigma^{i j} N\right)\left(\bar{\psi} \sigma^{k 0} \psi\right)\right],
\end{aligned}
$$

whose nonrelativistic limit for the nucleons, from the definitions (15), imply the following terms for the electron Lagrangian:

$$
\begin{aligned}
\mathcal{L}_{\mathrm{LVe}-3}= & \sum_{N}\left[\left(k_{A T}\right)_{i 0 j}\left(-\left\langle\sigma^{i}\right\rangle_{N}\right)\left(\bar{\psi} \sigma^{0 j} \psi\right)\right. \\
& +\left(k_{A T}\right)_{i j 0}\left(-\left\langle\sigma^{i}\right\rangle_{N}\right)\left(\bar{\psi} \sigma^{j 0} \psi\right) \\
& \left.+\left(k_{T A}\right)_{0 i j}\left(\epsilon_{i j k}\left\langle\sigma^{k}\right\rangle_{N}\right)\left(\bar{\psi} \gamma^{0} \gamma_{5} \psi\right)\right] \cdot n(\boldsymbol{r}),
\end{aligned}
$$

and

$$
\begin{aligned}
\mathcal{L}_{\mathrm{LVe}-4}= & \sum_{N}\left[\left(k_{T T}\right)_{i j 0 k}\left(\epsilon_{i j l}\left\langle\sigma^{l}\right\rangle_{N}\right)\left(\bar{\psi} \sigma^{0 k} \psi\right)\right. \\
& \left.+\left(k_{T T}\right)_{i j k 0}\left(\epsilon_{i j l}\left\langle\sigma^{l}\right\rangle_{N}\right)\left(\bar{\psi} \sigma^{k 0} \psi\right)\right] \cdot n(\boldsymbol{r}) .
\end{aligned}
$$

Below we will show how to read the EDM contributions from a $P$-odd and $T$-odd Hamiltonian piece.

\section{SPECTRUM SHIFTS AND EDMs}

In order to illustrate how $P$-odd and $T$-odd $e-N$ couplings generate EDM, we follow Refs. [4,18,24]. Consider the relativistic Hamiltonian of the valence electron under a radial potential, $\Phi_{\text {int }}(\boldsymbol{r})$,

$$
H_{0}=\boldsymbol{\alpha} \cdot \boldsymbol{p}+m \gamma^{0}-e \Phi_{\mathrm{int}}(\boldsymbol{r}),
$$

whose eigenstates are $\left|\psi_{0}\right\rangle$. If an external electric field, $\boldsymbol{E}=E_{z} \hat{z}$, is applied, the solutions, to first order in $\boldsymbol{E}$, become 


$$
|\Psi\rangle=\left|\psi_{0}\right\rangle+e E_{z}|\eta\rangle,
$$

where

$$
|\eta\rangle=\sum_{n \neq 0} \frac{\left|\psi_{n}\right\rangle\left\langle\psi_{n}|z| \psi_{0}\right\rangle}{E_{0}-E_{n}}
$$

We will use the data available in Ref. [24] for the thallium $(A=205$ and $Z=81)$ valence electron under a modified Tiez potential, $\Phi_{\text {int }}(\boldsymbol{r})$. The states $\left|\psi_{0}\right\rangle$ and $|\eta\rangle$ have opposite parity (the ground state $\left|\psi_{0}\right\rangle$ has $l=1$ and $|\eta\rangle$ has $l=0)$. The expressions for the unperturbed wave function $\psi_{0}($ with $l=1)$ and for the state $\eta$ (with $l=0$ ) are, respectively,

$$
\begin{gathered}
\left(\psi_{0}\right)_{J=\frac{1}{2}, m=\frac{1}{2}}^{l}=\left(\begin{array}{c}
\frac{i}{r} G_{l, J=\frac{1}{2}}(r) \phi_{\frac{1}{2}, \frac{1}{2}}^{l} \\
\frac{1}{r} F_{l, J=\frac{1}{2}}(r)(\boldsymbol{\sigma} \cdot \hat{\boldsymbol{r}}) \phi_{\frac{1}{2}, \frac{1}{2}}^{l}
\end{array}\right), \\
\eta_{J=\frac{1}{2}, m=\frac{1}{2}}^{l}=\left(\begin{array}{c}
\frac{i}{r} G_{l, J=\frac{1}{2}}^{S}(r) \phi_{\frac{1}{2}, \frac{1}{2}}^{l} \\
\frac{1}{r}\left(F_{l, J=\frac{1}{2}}^{S}(r) \boldsymbol{\sigma} \cdot \hat{\boldsymbol{r}}\right) \phi_{\frac{1}{2}, \frac{1}{2}}^{l}
\end{array}\right),
\end{gathered}
$$

where,

$$
\phi_{\frac{1}{2}, \frac{1}{2}}^{l=0}=\left(\begin{array}{c}
Y_{0}^{0} \\
0
\end{array}\right), \quad \phi_{\frac{1}{2}, \frac{1}{2}}^{l=1}=\left(\begin{array}{c}
\sqrt{\frac{1}{3}} Y_{1}^{0} \\
-\sqrt{\frac{2}{3}} Y_{1}^{1}
\end{array}\right)
$$

where $Y_{l}^{m}$ corresponds to the normalized spherical harmonics. The superscript $S$ stands for Sternheimer solutions, which hold for the Sternheimer equation, $\left(H_{0}-E_{0}\right)|\eta\rangle=z\left|\psi_{0}\right\rangle$-see Refs. [20,66]. While the unperturbed spinor is normalized to unity, $\eta_{J=\frac{1}{2}, m=\frac{1}{2}}^{l}$ is not. It is nevertheless required that $G_{l, J=\frac{1}{2}}^{S}(r), F_{l, J=\frac{1}{2}}^{S}(r) \rightarrow 0$ far from the origin. These solutions have been used, for instance, in evaluating enhancement factors of atomic EDMs and atomic polarizations-see [4,24,27].

In order to calculate the EDMs arising from the $e-N$ couplings, we start by extracting from the Lagrangian (16), related to the rank-1 LV tensor, the following Hamiltonian contributions

$$
H_{\mathrm{LV} e-1}=\left[-\left(k_{S V}\right)_{i} A \gamma^{0} \gamma^{i}-\left(k_{V P}\right)_{0} A i \gamma^{0} \gamma_{5}\right] n(\boldsymbol{r}) .
$$

The corresponding energy shift for a given $P$-odd and $T$-odd Hamiltonian piece $H_{P, T}$ is

$$
\Delta E=\left\langle\Psi\left|H_{P, T}\right| \Psi\right\rangle=2 e E_{z} \Re\left[\int \psi_{0}^{\dagger} H_{P, T} \eta d^{3} r\right] .
$$

where we used that $|\Psi\rangle=\left|\psi_{0}\right\rangle+e E_{z}|\eta\rangle$, remembering that $H_{P, T}$ is $P$-odd. From Eq. (34), the EDM magnitude is read as the factor multiplying the electric field on the right hand side, $\left|d_{\text {equiv }}\right|=2 e \Re\left[\left\langle\psi_{0}\left|H_{P, T}\right| \eta\right\rangle\right]$, that is,

$$
\frac{\Delta E}{E_{z}}=2 e \Re\left[\left\langle\psi_{0}\left|H_{P, T}\right| \eta\right\rangle\right] \equiv d_{\text {equiv }} .
$$

In this case, $d_{\text {equiv }}$ is an EDM-equivalent due to the $P$-odd and $T$-odd LV interactions. Applying this prescription to the Hamiltonian (33) and using the spinors (30) and (31), we notice that the contribution from $\left(k_{S V}\right)_{i}$ has no real part, so that it does not yield $d_{\text {equiv }}$, according to Eq. (35) and Eqs. (40)-(42). As for the term $\left(k_{V P}\right)_{0}$, its contribution is similar to the one arising from the scalar-pseudoscalar coupling shown in Eq. (1), that is

$$
\left|d_{1-\text { equiv }}^{(1)}\right|=2 e A\left(k_{V P}\right)_{0} n(r)\left|\Re\left[\left\langle\psi_{0}\left|\left(i \gamma^{0} \gamma_{5}\right)\right| \eta\right\rangle\right]\right|,
$$

which can be written as

$$
\begin{aligned}
\left|d_{1 \text {-equiv }}^{(1)}\right|= & 2 e\left(k_{V P}\right)_{0} \frac{3 A}{4 \pi R_{\text {Nucleus }}^{3}} \int_{0}^{R_{\text {Nucleus }}}\left[F^{S}(r) G(r)\right. \\
& \left.+G^{S}(r) F(r)\right] d r,
\end{aligned}
$$

where we used a uniform nucleon density, $n=$ $3 / 4 \pi R_{\text {Nucleus }}^{3}$. Supposing $P$-odd and $T$-odd $e-N$ couplings are the only source of atomic EDM, we can set bounds on $\left(k_{V P}\right)_{0}$ by using the data of the thallium atom, available in Ref. [24,67], and the current upper limit on the electron's EDM, $\left|\mathbf{d}_{e}\right|<1.1 \times 10^{-29} e \cdot \mathrm{cm}$ in Ref. [9]. This implies the following upper bound

$$
\left|\left(k_{V P}\right)_{0}\right|<1.6 \times 10^{-15}(\mathrm{GeV})^{-2} .
$$

Since the limit (38) holds for the zero component of the vector, it is not subjected to sidereal variations, unlike the ones attained in Refs. $[59,60]$. We must point out, however, that this term, being $C P T$-odd, also violates $C$, so that, unlike usual EDMs, it may play a role in the baryon asymmetry in the universe.

As for the Hamiltonian contribution arising from couplings with a rank-2 LV tensor

$$
H_{\mathrm{LVe}-2}=\left[-A\left(k_{V V}\right)_{0 i} \gamma^{0} \gamma^{i}-\left(k_{A A}\right)_{i 0}\left\langle\sigma^{i}\right\rangle_{N} \gamma_{5}\right] n(\boldsymbol{r}),
$$

we find that its pieces do not yield $d_{\text {equiv }}$, according to Eq. (35), for they generate imaginary contributions. In summary, for internal products of the form $\left\langle\psi_{0}|\mathcal{O}| \eta\right\rangle$, in which the operator $\mathcal{O}$ is a $4 \times 4$ matrix, the following results are useful:

$$
\left\langle\psi_{0}\left|\gamma^{0} \gamma^{i}\right| \eta\right\rangle=i \delta_{i 3} \int_{0}^{R_{\text {Nucleus }}}\left[\frac{1}{3} F^{S}(r) G(r)+G^{S}(r) F(r)\right] d r,
$$




$$
\begin{aligned}
& \left\langle\psi_{0}\left|\gamma_{5}\right| \eta\right\rangle=i \int_{0}^{R_{\text {Nucleus }}}\left[-F^{S}(r) G(r)+G^{S}(r) F(r)\right] d r, \\
& \left\langle\psi_{0}\left|i \gamma^{i}\right| \eta\right\rangle=\delta_{i 3} \int_{0}^{R_{\text {Nucleus }}}\left[-\frac{1}{3} F^{S}(r) G(r)+G^{S}(r) F(r)\right] d r,
\end{aligned}
$$

where the $\delta_{i 3}$ means that only the $i=3$ component survives, due to the structure of the $\gamma^{3}$ matrix, while the analogue contributions for $i=1,2$ vanish.

For the rank-3 and rank- 4 cases, the Hamiltonians are

$$
\begin{gathered}
H_{\mathrm{LVe}-3}=\left[\left(k_{A T}\right)_{i 0 j}\left\langle\sigma^{i}\right\rangle_{N} i \gamma^{0} \alpha^{j}-\left(k_{A T}\right)_{i j 0}\left\langle\sigma^{i}\right\rangle_{N} i \gamma^{0} \alpha^{j}\right. \\
\left.-\left(k_{T A}\right)_{0 i j} \epsilon_{i j k}\left\langle\sigma^{k}\right\rangle_{N} \gamma_{5}\right] \cdot n(\boldsymbol{r}), \\
H_{\mathrm{LVe}-4}=\left[-\left(k_{T T}\right)_{i j 0 k} \epsilon_{i j l}\left\langle\sigma^{l}\right\rangle_{N} i \gamma^{0} \alpha^{k}\right. \\
\left.+\left(k_{T T}\right)_{i j k 0} \epsilon_{i j l}\left\langle\sigma^{l}\right\rangle_{N} i \gamma^{0} \alpha^{k}\right] \cdot n(\boldsymbol{r}),
\end{gathered}
$$

in which the term $\left(k_{T A}\right)_{0 i j}$, according to Eq. (41), yields no real contribution. Concerning the remaining pieces, the Hamiltonian (44) can also be read as

$$
H_{\mathrm{LVe}-4}=\left[-\left(K_{T T}\right)_{0 k l}\left\langle\sigma^{l}\right\rangle_{N} i \gamma^{k}+\left(K_{T T}\right)_{0 k l}\left\langle\sigma^{l}\right\rangle_{N} i \gamma^{k}\right] \cdot n(\boldsymbol{r}),
$$

with the redefinition $\left(k_{T T}\right)_{i j 0 k} \epsilon_{i j l}=\left(K_{T T}\right)_{0 k l}$. The EDM contributions from the rank-3 Hamiltonian are

$$
\begin{gathered}
d_{3-\text { equiv }}^{(1)}=2 e\left(k_{A T}\right)_{i 03}\left\langle\sigma^{i}\right\rangle_{N} \Re\left[\left\langle\psi_{0}\left|i \gamma^{3}\right| \eta\right\rangle\right], \\
d_{3-\text { equiv }}^{(2)}=-2 e\left(k_{A T}\right)_{i 30}\left\langle\sigma^{i}\right\rangle_{N} \Re\left[\left\langle\psi_{0}\left|i \gamma^{3}\right| \eta\right\rangle\right],
\end{gathered}
$$

and from rank-4,

$$
\begin{aligned}
d_{4-\text { equiv }}^{(1)} & =-2 e\left(K_{T T}\right)_{03 l}\left\langle\sigma^{l}\right\rangle_{N} \Re\left[\left\langle\psi_{0}\left|i \gamma^{3}\right| \eta\right\rangle\right], \\
d_{4-\text { equiv }}^{(2)} & =2 e\left(K_{T T}\right)_{30 l}\left\langle\sigma^{l}\right\rangle_{N} \Re\left[\left\langle\psi_{0}\left|i \gamma^{3}\right| \eta\right\rangle\right] .
\end{aligned}
$$

We point out that the presence of the factor $\epsilon_{i j k}$ in Eq. (44) induces an effect similar to a rotation on the nucleon's spin $\left\langle\sigma^{l}\right\rangle_{N}$ or background vector, coupling orthogonal components of $\left\langle\sigma^{l}\right\rangle_{N}$ and $\left(k_{T T}\right)_{i j 03}$. Accordingly, say we pick $i=1$, then $j=2$, and we must use a nucleon in the spin state $\left\langle\sigma^{z}\right\rangle_{N}$ in order to obtain the EDM contribution arising from $\left(k_{T T}\right)_{1203}$, which corresponds to constrain $\left(K_{T T}\right)_{033}$.

Assuming that the thallium valence nucleon has spin $\left\langle\sigma^{z}\right\rangle_{N}= \pm 1$ [67], and that the atomic integral in Eq. (42) has the same magnitude as the one in Eq. (37), we attain, for the rank-3 couplings, the upper bounds

$$
\left|\left(k_{A T}\right)_{i 03}\right|<3.2 \times 10^{-13}(\mathrm{GeV})^{-2},
$$

$$
\left|\left(k_{A T}\right)_{i 30}\right|<3.2 \times 10^{-13}(\mathrm{GeV})^{-2},
$$

and for the rank-4:

$$
\begin{aligned}
& \left|\left(K_{T T}\right)_{03 l}\right|<3.2 \times 10^{-13}(\mathrm{GeV})^{-2}, \\
& \left|\left(K_{T T}\right)_{30 l}\right|<3.2 \times 10^{-13}(\mathrm{GeV})^{-2} .
\end{aligned}
$$

In these cases, the bounds suffer sidereal variations, for these are in fact measured in the Lab's reference frame, in which these tensor components are not constant. This issue will be addressed in the Sec. III A while the constraints are summarized in Table V.

\section{A. Sidereal analysis}

Because the LV background tensors are constant only in an inertial reference frame (RF), such as the Sun's rest frame, it is necessary, therefore, to show how to translate these bounds to the Earth-located Lab's RF, at the colatitude $\chi$, rotating around the Earth's axis with angular velocity $\Omega$. In short, the bounds should be written in terms of the tensor components in the Sun's RF. For experiments up to a few weeks long, the transformation law for a rank-2 tensor, say $B_{\mu \nu}$, according to Refs. $[59,68]$, is merely a spatial rotation,

$$
B_{\mu \nu}^{(\mathrm{Lab})}=\mathcal{R}_{\mu \alpha} \mathcal{R}_{\nu \beta} B_{\alpha \beta}^{(\mathrm{Sun})},
$$

where

$$
R_{\mu \nu}=\left(\begin{array}{cccc}
1 & 0 & 0 & 0 \\
0 & \cos \chi \cos \Omega t & \cos \chi \sin \Omega t & -\sin \chi \\
0 & -\sin \Omega t & \cos \Omega t & 0 \\
0 & \sin \chi \cos \Omega t & \sin \chi \sin \Omega t & \cos \chi
\end{array}\right),
$$

in which the first line and column were included for completeness. According to the transformation law (54), the components $\left(k_{A T}\right)_{i 03}$ and $\left(k_{A T}\right)_{i 30}$ transform as

$$
\begin{aligned}
& \left(k_{A T}\right)_{i 03}^{(\mathrm{Lab})}=\mathcal{R}_{i k} \mathcal{R}_{3 l}\left(k_{A T}\right)_{k 0 l}^{(\mathrm{Sun})}, \\
& \left(k_{A T}\right)_{i 30}^{(\mathrm{Lab})}=\mathcal{R}_{i k} \mathcal{R}_{3 l}\left(k_{A T}\right)_{k l 0}^{(\mathrm{Sun})},
\end{aligned}
$$

whose time averages for $l=1,2,3$ yield, respectively:

$$
\begin{aligned}
\left\langle\left(k_{A T}\right)_{103}^{(\mathrm{Lab})}\right\rangle & =\frac{1}{4}\left[\left(k_{A T}\right)_{101}^{(\mathrm{Sun})}+\left(k_{A T}\right)_{202}^{(\mathrm{Sun})}-2\left(k_{A T}\right)_{303}^{(\mathrm{Sun})}\right] \sin 2 \chi \\
\left\langle\left(k_{A T}\right)_{203}^{(\mathrm{Lab})}\right\rangle= & {\left[-\left(k_{A T}\right)_{102}^{(\text {Sun })}+\left(k_{A T}\right)_{201}^{(\mathrm{Sun})}\right] \sin \chi } \\
\left\langle\left(k_{A T}\right)_{303}^{(\mathrm{Lab})}\right\rangle= & {\left[\frac{1}{2}\left(\left(k_{A T}\right)_{101}^{(\mathrm{Sun})}+\left(k_{A T}\right)_{202}^{(\mathrm{Sun})}\right) \sin ^{2} \chi\right.} \\
& \left.+\left(k_{A T}\right)_{303}^{(\text {Sun })} \cos ^{2} \chi\right]
\end{aligned}
$$


TABLE V. Bounds on the LV tensors of ranks ranging from 1 to 4.

\begin{tabular}{|c|c|}
\hline Component & Upper bound \\
\hline$\left|\left(k_{V P}\right)_{0}^{(\mathrm{Sun})}\right|$ & $1.6 \times 10^{-15}(\mathrm{GeV})^{-2}$ \\
\hline$\frac{1}{4}\left[\left(k_{A T}\right)_{101}^{(\text {Sun })}+\left(k_{A T}\right)_{202}^{(\text {Sun })}-2\left(k_{A T}\right)_{303}^{(\text {Sun })}\right] \sin 2 \chi \mid$ & $3.2 \times 10^{-13}(\mathrm{GeV})^{-2}$ \\
\hline$\left[-\left(k_{A T}\right)_{102}^{(\text {Sun })}+\left(k_{A T}\right)_{201}^{(\text {Sun })}\right] \sin \chi \mid$ & $3.2 \times 10^{-13}(\mathrm{GeV})^{-2}$ \\
\hline$\left[\frac{1}{2}\left(\left(k_{A T}\right)_{101}^{(\text {Sun })}+\left(k_{A T}\right)_{202}^{(\text {Sun })}\right) \sin ^{2} \chi+\left(k_{A T}\right)_{303}^{(\text {Sun })} \cos ^{2} \chi\right]$ & $3.2 \times 10^{-13}(\mathrm{GeV})^{-2}$ \\
\hline$\frac{1}{4}\left[\left(k_{A T}\right)_{110}^{(\text {Sun })}+\left(k_{A T}\right)_{220}^{(\text {Sun })}-2\left(k_{A T}\right)_{330}^{(\text {Sun })}\right] \sin 2 \chi \mid$ & $3.2 \times 10^{-13}(\mathrm{GeV})^{-2}$ \\
\hline$\left[-\left(k_{A T}\right)_{120}^{(\text {Sun })}+\left(k_{A T}\right)_{210}^{(\text {Sun })}\right] \sin \chi \mid$ & $3.2 \times 10^{-13}(\mathrm{GeV})^{-2}$ \\
\hline$\left[\frac{1}{2}\left(\left(k_{A T}\right)_{110}^{(\text {Sun })}+\left(k_{A T}\right)_{220}^{(\text {Sun })}\right) \sin ^{2} \chi+\left(k_{A T}\right)_{330}^{(\text {Sun })} \cos ^{2} \chi\right]$ & $3.2 \times 10^{-13}(\mathrm{GeV})^{-2}$ \\
\hline$\frac{1}{4}\left[\left(K_{T T}\right)_{011}^{(\text {Sun })}+\left(K_{T T}\right)_{022}^{(\text {Sun })}-2\left(K_{T T}\right)_{033}^{(\text {Sun })}\right] \sin 2 \chi \mid$ & $3.2 \times 10^{-13}(\mathrm{GeV})^{-2}$ \\
\hline$\left[\left(K_{T T}\right)_{012}^{(\text {Sun })}-\left(K_{T T}\right)_{021}^{(\text {Sun })}\right] \sin \chi \mid$ & $3.2 \times 10^{-13}(\mathrm{GeV})^{-2}$ \\
\hline$\left[\frac{1}{2}\left(\left(K_{T T}\right)_{011}^{(\text {Sun })}+\left(K_{T T}\right)_{022}^{(\text {Sun })}\right) \sin ^{2} \chi+\left(K_{T T}\right)_{033}^{(\text {Sun })} \cos ^{2} \chi\right]$ & $3.2 \times 10^{-13}(\mathrm{GeV})^{-2}$ \\
\hline$\frac{1}{4}\left[\left(K_{T T}\right)_{101}^{(\text {Sun })}+\left(K_{T T}\right)_{202}^{(\text {Sun })}-2\left(K_{T T}\right)_{303}^{(\text {Sun })}\right] \sin 2 \chi \mid$ & $3.2 \times 10^{-13}(\mathrm{GeV})^{-2}$ \\
\hline$\left|\left[\left(K_{T T}\right)_{102}^{(\text {Sun })}-\left(K_{T T}\right)_{201}^{(\text {Sun })}\right] \sin \chi\right|$ & $3.2 \times 10^{-13}(\mathrm{GeV})^{-2}$ \\
\hline$\left[\frac{1}{2}\left(\left(K_{T T}\right)_{101}^{(\text {Sun })}+\left(K_{T T}\right)_{202}^{(\text {Sun })}\right) \sin ^{2} \chi+\left(K_{T T}\right)_{303}^{(\text {Sun })} \cos ^{2} \chi\right]$ & $3.2 \times 10^{-13}(\mathrm{GeV})^{-2}$ \\
\hline
\end{tabular}

and similar expressions for $\left(k_{A T}\right)_{130}^{(\mathrm{Lab})},\left(k_{A T}\right)_{230}^{(\mathrm{Lab})}$, and $\left(k_{A T}\right)_{330}^{(\mathrm{Lab})}$. The same procedure can be applied to the components of $\left(K_{T T}\right)_{03 l}$ for $l=1,2$, 3, yielding, respectively,

$$
\begin{aligned}
\left\langle\left(K_{T T}\right)_{031}^{(\mathrm{Lab})}\right\rangle= & \frac{1}{4}\left[\left(K_{T T}\right)_{011}^{(\mathrm{Sun})}+\left(K_{T T}\right)_{022}^{(\mathrm{Sun})}\right. \\
& \left.-2\left(K_{T T}\right)_{033}^{(\text {Sun })}\right] \sin 2 \chi \\
\left\langle\left(K_{T T}\right)_{032}^{(\mathrm{Lab})}\right\rangle= & {\left[-\left(K_{T T}\right)_{012}^{(\text {Sun })}+\left(K_{T T}\right)_{201}^{(\mathrm{Sun})}\right] \sin \chi } \\
\left\langle\left(K_{T T}\right)_{303}^{(\mathrm{Lab})}\right\rangle= & {\left[\frac{1}{2}\left(\left(K_{T T}\right)_{101}^{(\text {Sun })}+\left(K_{T T}\right)_{202}^{(\text {Sun })}\right) \sin ^{2} \chi\right.} \\
& \left.+\left(K_{T T}\right)_{303}^{(\text {Sun })} \cos ^{2} \chi\right],
\end{aligned}
$$

and similar expressions for the components $\left(K_{T T}\right)_{30 l}$. Finally, the sidereal variations of the bounds (52) are displayed as:

$$
\begin{aligned}
& \left|\frac{1}{4}\left[\left(K_{T T}\right)_{011}^{(\text {Sun })}+\left(K_{T T}\right)_{022}^{(\text {Sun })}-2\left(K_{T T}\right)_{033}^{(\text {Sun })}\right] \sin 2 \chi\right| \\
& <3.2 \times 10^{-13}(\mathrm{GeV})^{-2} \\
& \left|\left[\left(K_{T T}\right)_{012}^{(\text {Sun })}-\left(K_{T T}\right)_{021}^{(\text {Sun })}\right] \sin \chi\right| \\
& <3.2 \times 10^{-13}(\mathrm{GeV})^{-2} \\
& \left|\left[\frac{1}{2}\left(\left(K_{T T}\right)_{011}^{(\text {Sun })}+\left(K_{T T}\right)_{022}^{(\text {Sun })}\right) \sin ^{2} \chi+\left(K_{T T}\right)_{033}^{(\text {Sun })} \cos ^{2} \chi\right]\right| \\
& <3.2 \times 10^{-13}(\mathrm{GeV})^{-2},
\end{aligned}
$$

For clarity, the bounds (50)-(53), in terms of the Sun's RF quantities, are listed in the Table $\mathrm{V}$ for $l=1,2,3$.

\section{CONCLUSION AND FINAL REMARKS}

One point to be noted is that the 4-fermion couplings here presented involve two kinds of fermion spinors, that is, we consider both $\left(k_{S V}\right)_{\mu}(\bar{N} N)\left(\bar{\psi} \gamma^{\mu} \psi\right)$ and $\left(k_{V S}\right)_{\mu}\left(\bar{N} \gamma^{\mu} N\right)(\bar{\psi} \psi)$. Such a distinction is not important when one does not differ between the interacting fermion bilinears, but it should be taken into account for dimension6 electron-nucleon interactions. Accordingly, the couplings presented in Tables I and IV are displayed in Ref. [65], the only difference is that here we consider twice as many couplings, since we are dealing with different spinors. To illustrate the redundancies, we have included comments and the couplings in Table II, which are particular parametrizations of the ones of rank-3 listed in Table IV.

Still concerning Ref. [65], it is worth mentioning that the first line of the dimension- 6 couplings of its Table III contains

$$
\begin{aligned}
& \left(k_{S S}\right)(\bar{N} N)(\bar{\psi} \psi), \quad\left(k_{P P}\right)\left(\bar{N} i \gamma_{5} N\right)\left(\bar{\psi} i \gamma_{5} \psi\right), \\
& \left(k_{S P}\right)(\bar{N} N)\left(\bar{\psi} i \gamma_{5} \psi\right), \quad\left(k_{P S}\right)\left(\bar{N} i \gamma_{5} N\right)(\bar{\psi} \psi),
\end{aligned}
$$

that do not violate Lorentz symmetry. From those, only the last two are $P$-odd and $T$-odd and generate EDM, and only the third one is not suppressed, being equivalent to 


$$
\mathcal{L}_{\psi \psi}^{(6)}=i\left(\kappa_{S P}\right)(\bar{\psi} \psi)\left(\bar{\psi} \gamma^{5} \psi\right)
$$

Such a coupling is identical to the standard scalarpseudoscalar electron-nucleon interaction in Eq. (1) $[4,14,24]$. It yields

$$
\begin{aligned}
d_{\text {equiv }-\kappa_{S P}}= & 2 e A \kappa_{S P} \frac{3}{4 \pi R_{\text {Nucleus }}^{3}} \int_{0}^{R_{\text {Nucleus }}}\left(F^{S}(r) G(r)\right. \\
& \left.+G^{S}(r) F(r)\right) d r,
\end{aligned}
$$

and must fulfill

$$
\kappa_{S P}<1.6 \times 10^{-15}(\mathrm{GeV})^{-2} .
$$

Clearly, due to the scalar form of the coupling (62), the coefficient $\kappa_{S P}$ does not suffer sidereal variations.

In outlining the procedure, we stress that the possibilities of couplings with LV tensors of ranks ranging from 1 to 4 were listed in Tables I and IV. Their behavior under $C, P$ and $T$ was crucial to extract the components compatible with EDM generation. After applying the nonrelativistic limit for the nucleons, a few candidates were suppressed and no longer considered. The remaining unsuppressed couplings had their Hamiltonian pieces evaluated in Eqs. (33), (39), (43), and (44), then their respective EDM-equivalent contributions were calculated as in Eq. (35), via atomic parity nonconservation methods. Also, as mentioned in Sec. II, due to their matrix structure, some couplings yield no real contribution to the energy shift, i.e., null $d_{\text {equiv }}$, being then discarded. The surviving terms had their magnitudes limited using the electron's
EDM data, which is justified by the fact that the measured atomic EDM (attributed to either an unpaired electron or nucleon, depending on the experiment) may be due to $P$-odd and $T$-odd $e-N$ interactions as well-thus, we have just chosen the most restrictive upper bound.

The most stringent upper bounds of $1.6 \times 10^{-15}(\mathrm{GeV})^{-2}$, in Eqs. (38) and (64), were set on the terms proportional to the atomic mass $A$, i.e., the terms $\left(k_{V P}\right)_{0}$ and $\kappa_{S P}$-these are the dominant contributions. The other bounds are about 2 orders of magnitude less restrictive, due to the fact that the nucleons' spins do not add up coherently. Moreover, the bounds with spatial indices suffer sidereal variations due to the Earth's rotation and Lab's location, and were transformed according to the rule (54) and then time averaged, providing the bounds in Table V.

\section{ACKNOWLEDGMENTS}

The authors are grateful to CNPq, CAPES, FAPESP, and FAPEMA (Brazilian research agencies) for invaluable financial support. We thank Dr. Nodoka Yamanaka and Dr. Alan Kostelecky for the useful and relevant feedback and private communications. We also thank Dr. Paul Mansfield and João A. A. de Simões, for the interesting discussions. This study was financed in part by the Coordenação de Aperfeiçoamento de Pessoal de Nível SuperiorBrazil (CAPES)—Finance Code 001, and by FCT Portugal Ref. UID/FIS/04564/2016. M. S. thanks CNPq [303482/2017-6]. M. M. F. is also grateful to FAPEMA/ UNIVERSAL/00880/15; FAPEMA/PRONEX 01452-14; CNPq/PRODUTIVIDADE/308933/2015-0.
[1] W. Bernreuther and M. Suzuki, Rev. Mod. Phys. 63, 313 (1991).

[2] J. S. M. Ginges and V. V. Flambaum, Phys. Rep. 397, 63 (2004); Phys. Rev. A 65, 032113 (2002); J. Jesus and J. Engel, Phys. Rev. C 72, 045503 (2005).

[3] J. Engel, M. J. Ramsey-Musolf, and U. van Kolck, Prog. Part. Nucl. Phys. 71, 21 (2013); T. Chupp and M. Ramsey-Musolf, Phys. Rev. C 91, 035502 (2015).

[4] B. Lee Roberts and W. J. Marciano, Lepton Dipole Moments, Advanced Series on Directions in High Energy Physics (Word Scientific, Singapore, 2010).

[5] N. Yamanaka, Int. J. Mod. Phys. E 26, 1730002 (2017); N. Yamanaka, B. Sahoo, N. Yoshinaga, T. Sato, K. Asahi, and B. Das, Eur. Phys. J. A 53, 54 (2017).

[6] M. Pospelov and A. Ritz, Ann. Phys. (Amsterdam) 318, 119 (2005).

[7] J. P. Lees et al. (BABAR Collaboration), Phys. Rev. Lett. 109, 211801 (2012).
[8] A. D. Sakharov, Pis'ma Zh. Eksp. Teor. Fiz. 5, 32 (1967) [JETP Lett. 5, 24 (1967)].

[9] ACME Collaboration, Nature (London) 562, 355 (2018).

[10] R. Engfer and H. K. Walter, Annu. Rev. Nucl. Part. Sci. 36, 327 (1986).

[11] B. C. Regan, E. D. Commins, C. J. Schmidt, and D. DeMille, Phys. Rev. Lett. 88, 071805 (2002); J. J. Hudson, D. M. Kara, I. J. Smallman, B. E. Sauer, M. R. Tarbutt, and E. A. Hinds, Nature (London) 473, 493 (2011); D. M. Kara, I. J. Smallman, J. J. Hudson, B. E. Sauer, M. R Tarbutt, and E. A. Hinds, New J. Phys. 14, 103051 (2012).

[12] J. Baron et al. (ACME Collaboration), Science 343, 269 (2014).

[13] N. Cabibbo, Phys. Rev. Lett. 10, 531 (1963); M. Pospelov and I. B. Khriplovich, Sov. J. Nucl. Phys. 53, 638 (1991).

[14] M. Pospelov and A. Ritz, Phys. Rev. D 89, 056006 (2014).

[15] O. P. Sushkov, V. V. Flambaum, and I. B. Khriplovich, Zh. Eksp. Teor. Fiz. 87, 1521 (1984) [Sov. Phys. JETP 60, 873 
(1984)]; V. V. Flambaum, I. B. Khriplovich, and O.P. Sushkov, Nucl. Phys. A449, 750 (1986).

[16] I. I. Schiff, Phys. Rev. 132, 2194 (1963).

[17] P. G. H. Sandars, J. Phys. B 1, 499 (1968).

[18] P. G. H. Sandars, J. Phys. B 1, 511 (1968).

[19] P. G. H. Sandars, Phys. Lett. 14, 194 (1965).

[20] P. G. H. Sandars and R. M. Sternheimer, Phys. Rev. A 11, 473 (1975).

[21] B. Graner, Y. Chen, E. G. Lindahl, and B. R. Heckel, Phys. Rev. Lett. 116, 161601 (2016).

[22] J. E. Kim, Phys. Rep. 150, 1 (1987); H.-Y. Cheng, Phys. Rep. 158, 1 (1988); J. E. Kim and G. Carosi, Rev. Mod. Phys. 82, 557 (2010); G. Pignol, Int. J. Mod. Phys. A 30, 1530048 (2015); Y. V. Stadnik and V. V. Flambaum, Phys. Rev. D 89, 043522 (2014); B. M. Roberts, Y. V. Stadnik, V. A. Dzuba, V. V. Flambaum, N. Leefer, and D. Budker, Phys. Rev. D 90, 096005 (2014).

[23] S. M. Barr, Phys. Rev. D 45, 4148 (1992).

[24] B. Bederson and H. Walther, Advances in Atomic, Molecular and Optical Physics (Academic Press, London, 1999), Vol. 40.

[25] M. A. Bouchiat and C. C. Bouchiat, Phys. Lett. B48, 111 (1974).

[26] I. B. Khriplovich and S. K. Lamoreaux, CP Violation Without Strangeness (Springer, Berlin, 1997).

[27] C. Bouchiat, Phys. Lett. 57B, 284 (1975); E. A. Hinds, C. E. Loving, and G. P. H. Sandars, Phys. Lett. 62B, 97 (1976); A. M. Martensson-Pendrill, Phys. Rev. Lett. 54, 1153 (1985); V. A. Dzuba, V. V. Flambaum, and P. G. Silvestrov, Phys. Lett. 154B, 93 (1985); X.-G. He and B. McKellar, Phys. Lett. B 390, 318 (1997).

[28] D. V. Neuffer and E. D. Commins, Phys. Rev. A 16, 844 (1977); 16, 1760 (1977); W. R. Johnson, Phys. Scr. 36, 765 (1987).

[29] N. Yamanaka, Phys. Rev. D 85, 115012 (2012); K. Yanase, N. Yoshinaga, K. Higashiyama, and N. Yamanaka, Phys. Rev. D 99, 075021 (2019).

[30] V. Alan Kostelecky, Phys. Rev. D 69, 105009 (2004); D. Colladay and V. A. Kostelecky, Phys. Rev. D 55, 6760 (1997); D. Colladay and V. A. Kostelecky, Phys. Rev. D 58, 116002 (1998); S. R. Coleman and S. L. Glashow, Phys. Rev. D 59, 116008 (1999).

[31] V. A. Kostelecky and C. D. Lane, J. Math. Phys. 40, 6245 (1999); R. Lehnert, J. Math. Phys. 45, 3399 (2004); D. Colladay and V. A. Kostelecky, Phys. Lett. B 511, 209 (2001); O. G. Kharlanov and V. Ch. Zhukovsky, J. Math. Phys. 48, 092302 (2007); R. Lehnert, Phys. Rev. D 68, 085003 (2003); V. A. Kostelecky and R. Lehnert, Phys. Rev. D 63, 065008 (2001); S. Chen, B. Wang, and R. Su, Classical Quantum Gravity 23, 7581 (2006); B. Gonçalves, M. M. Dias Junior, and B. J. Ribeiro, Phys. Rev. D 90, 085026 (2014).

[32] R. Bluhm, V. A. Kostelecky, and N. Russell, Phys. Rev. Lett. 79, 1432 (1997); Phys. Rev. D 57, 3932 (1998); Phys. Rev. Lett. 82, 2254 (1999); R. Bluhm, V. A. Kostelecky, C. D. Lane, and N. Russell, Phys. Rev. Lett. 88, 090801 (2002); R. Bluhm and V. A. Kostelecky, Phys. Rev. Lett. 84, 1381 (2000); R. Bluhm, V. A. Kostelecky, and C. D. Lane, Phys.
Rev. Lett. 84, 1098 (2000); V. A. Kostelecky and C. D. Lane, Phys. Rev. D 60, 116010 (1999).

[33] T. Mariz, J. R. Nascimento, and A. Yu. Petrov, Phys. Rev. D 85, 125003 (2012); A. P. Baeta Scarpelli, Marcos Sampaio, M. C. Nemes, and B. Hiller, Eur. Phys. J. C 56, 571 (2008); F. A. Brito, L. S. Grigorio, M. S. Guimaraes, E. Passos, and C. Wotzasek, Phys. Rev. D 78, 125023 (2008); F. A. Brito, E. Passos, and P. V. Santos, Europhys. Lett. 95, 51001 (2011); C. F. Farias, A. C. Lehum, J. R. Nascimento, and A. Yu. Petrov, Phys. Rev. D 86, 065035 (2012); J. R. Nascimento, A. Yu. Petrov, C. Wotzasek, and C. A. D. Zarro, Phys. Rev. D 89, 065030 (2014).

[34] R. V. Maluf, J. E. G. Silva, W. T. Cruz, and C. A. S. Almeida, Phys. Lett. B 738, 341 (2014); B. Gonçalves, M. M. Dias, Jr., and B. J. Ribeiro, Phys. Rev. D 99, 096015 (2019); Z. Xiao, Phys. Rev. D 94, 115020 (2016); Z. Xiao, Phys. Rev. D 93, 125022 (2016); Z. Xiao and Bo-Qiang Ma, Int. J. Mod. Phys. A 24, 1359 (2009).

[35] V. A. Kostelecky and M. Mewes, Phys. Rev. Lett. 87, 251304 (2001); V. A. Kostelecky and M. Mewes, Phys. Rev. D 66, 056005 (2002); V. A. Kostelecky and M. Mewes, Phys. Rev. Lett. 97, 140401 (2006); C. A. Escobar and M. A. G. Garcia, Phys. Rev. D 92, 025034 (2015); A. Martín-Ruiz and C. A. Escobar, Phys. Rev. D 94, 076010 (2016).

[36] S. M. Carroll, G. B. Field, and R. Jackiw, Phys. Rev. D 41, 1231 (1990); C. Adam and F. R. Klinkhamer, Nucl. Phys. B607, 247 (2001); B657, 214 (2003); Y. M. P. Gomes and P. C. Malta, Phys. Rev. D 94, 025031 (2016).

[37] A. P. Baeta Scarpelli, H. Belich, J. L. Boldo, and J. A. Helayel-Neto, Phys. Rev. D 67, 085021 (2003); L. H. C. Borges, F. A. Barone, and J. A. Helayel-Neto, Eur. Phys. J. C 74, 2937 (2014); T. R. S. Santos and R. F. Sobreiro, Phys. Rev. D 91, 025008 (2015); T. R. S. Santos and R.F. Sobreiro, Braz. J. Phys. 46, 437 (2016).

[38] T. R. S. Santos, R. F. Sobreiro, and A. A. Tomaz, Phys. Rev. D 94, 085027 (2016); Tiago R. S. Santos and Rodrigo F. Sobreiro, Eur. Phys. J. C 77, 903 (2017).

[39] O. G. Kharlanov and V. Ch. Zhukovsky, Phys. Rev. D 81, 025015 (2010); A. Martín-Ruiz and C. A. Escobar, Phys. Rev. D 94, 076010 (2016); B. Cruz, E. R. Bezerra de Mello, and A. Yu. Petrov, Phys. Rev. D 96, 045019 (2017); C. A. Escobar, J. Phys. Conf. Ser. 952, 012010 (2018); A. Martín-Ruiz and C. A. Escobar, Phys. Rev. D 95, 036011 (2017).

[40] F. R. Klinkhamer and M. Schreck, Nucl. Phys. B848, 90 (2011); M. Schreck, Phys. Rev. D 86, 065038 (2012); M. A. Hohensee, R. Lehnert, D. F. Phillips, and R. L. Walsworth, Phys. Rev. D 80, 036010 (2009); A. Moyotl, H. Novales-Sánchez, J. J. Toscano, and E. S. Tututi, Int. J. Mod. Phys. A 29, 1450039 (2014); 29, 1450107 (2014); M. Cambiaso, R. Lehnert, and R. Potting, Phys. Rev. D 90, 065003 (2014); R. Bufalo, Int. J. Mod. Phys. A 29, 1450112 (2014); G. P. de Brito, P. C. Malta, and L. P. R. Ospedal, Phys. Rev. D 95, 016006 (2017).

[41] G. P. de Brito, J. T. Guaitolini, Jr., D. Kroff, P. C. Malta, and C. Marques, Phys. Rev. D 94, 056005 (2016); T. R. S. Santos and R. F. Sobreiro, Phys. Rev. D 94, 125020 (2016); Adv. High Energy Phys. 2018, 4596129 (2018); 
F. E. P. dos Santos and M. M. Ferreira, Jr., Symmetry 10, 302 (2018); T. P. Netto, Phys. Rev. D 97, 055048 (2018); J. R. Nascimento, A. Yu. Petrov, and Carlos M. Reyes, Eur. Phys. J. C 78, 541 (2018).

[42] J. P. Noordmans, H. W. Wilschut, and R. G. E. Timmermans, Phys. Rev. C 87, 055502 (2013); Phys. Rev. Lett. 111, 171601 (2013); B. Altschul, Phys. Rev. D 87, 096004 (2013); Phys. Rev. D 88, 076015 (2013); J. P. Noordmans and K. K. Vos, Phys. Rev. D 89, 101702(R) (2014); J. S. Diaz, V. A. Kostelecky, and R. Lehnert, Phys. Rev. D 88, 071902(R) (2013); J. S. Diaz, Adv. High Energy Phys. 2014, 305298 (2014); K. K. Vos, H. W. Wilschut, and R. G. E. Timmermans, Phys. Rev. C 91, 038501 (2015); 92, 052501 (R) (2015); Rev. Mod. Phys. 87, 1483 (2015).

[43] J. Castro-Medina, H. Novales-Sanchez, and J. J. Toscano, Int. J. Mod. Phys. A 30, 1550216 (2015); M. A. LópezOsorio, E. Martínez-Pascual, and J. J. Toscano, J. Phys. G 43, 025003 (2016); J. I. Aranda, F. Ramirez-Zavaleta, D. A. Rosete, F. J. Tlachino, J. J. Toscano, and E. S. Tututi, Int. J. Mod. Phys. A 29, 1450180 (2014).

[44] D. Colladay, J. P. Noordmans, and R. Potting, Symmetry 9 , 248 (2017); Phys. Rev. D 96, 035034 (2017); J. Phys. Conf. Ser. 952, 012021 (2018); C. A. Escobar, J. P. Noordmans, and R. Potting, Phys. Rev. D 97, 115030 (2018); A. I. Hernández-Juárez, J. Montaño, H. Novales-Sánchez, M. Salinas, J. J. Toscano, and O. Vázquez-Hernández, Phys. Rev. D 99, 013002 (2019).

[45] V. A. Kostelecký and M. Mewes, Phys. Rev. D 80, 015020 (2009); M. Mewes, Phys. Rev. D 85, 116012 (2012); M. Schreck, Phys. Rev. D 89, 105019 (2014).

[46] V. A. Kostelecký and M. Mewes, Phys. Rev. D 88, 096006 (2013); M. Schreck, Phys. Rev. D 90, 085025 (2014); J. A. A. S. Reis and M. Schreck, Phys. Rev. D 95, 075016 (2017).

[47] R. C. Myers and M. Pospelov, Phys. Rev. Lett. 90, 211601 (2003); C. M. Reyes, L. F. Urrutia, and J. D. Vergara, Phys. Rev. D 78, 125011 (2008); Phys. Lett. B 675, 336 (2009); C. M. Reyes, Phys. Rev. D 82, 125036 (2010); Phys. Rev. D 80, 105008 (2009); Phys. Rev. D 87, 125028 (2013); C. M. Reyes, S. Ossandon, and C. Reyes, Phys. Lett. B 746, 190 (2015); A. Celeste, T. Mariz, J. R. Nascimento, and A. Yu. Petrov, Phys. Rev. D 93, 065012 (2016).

[48] M. Cambiaso, R. Lehnert, and R. Potting, Phys. Rev. D 85, 085023 (2012); B. Agostini, F. A. Barone, F. E. Barone, P. Gaete, and J. A. Helayël-Neto, Phys. Lett. B 708, 212 (2012); L. Campanelli, Phys. Rev. D 90, 105014 (2014); R. Bufalo, B. M. Pimentel, and D. E. Soto, Phys. Rev. D 90, 085012 (2014).

[49] Y. Ding and V. A. Kostelecky, Phys. Rev. D 94, 056008 (2016).

[50] H. Belich, T. Costa-Soares, M. M. Ferreira, Jr., and J. A. Helayël-Neto, Eur. Phys. J. C 41, 421 (2005); H. Belich, L. P. Colatto, T. Costa-Soares, J. A. Helayël-Neto, and M. T. D. Orlando, Eur. Phys. J. C 62, 425 (2009);
B. Charneski, M. Gomes, R. V. Maluf, and A. J. da Silva, Phys. Rev. D 86, 045003 (2012); A. F. Santos and Faqir C. Khanna, Phys. Rev. D 95, 125012 (2017).

[51] G. Gazzola, H. G. Fargnoli, A. P. Baeta Scarpelli, M. Sampaio, and M.C. Nemes, J. Phys. G 39, 035002 (2012); A. P. Baeta Scarpelli, J. Phys. G 39, 125001 (2012); L. C. T. Brito, H. G. Fargnoli, and A. P. Baeta Scarpelli, Phys. Rev. D 87, 125023 (2013); K. Bakke, H. Belich, and E. O. Silva, J. Math. Phys. 52, 063505 (2011); J. Phys. G 39, 055004 (2012); Ann. Phys. (Amsterdam) 523, 910 (2011); Y. M. P. Gomes and J. T. Guaitolini, Jr, Phys. Rev. D 99, 055006 (2019).

[52] L. H. C. Borges, A. G. Dias, A. F. Ferrari, J. R. Nascimento, and A. Yu. Petrov, Phys. Rev. D 89, 045005 (2014); L. H. C. Borges, A. G. Dias, A. F. Ferrari, J. R. Nascimento, and A. Yu. Petrov, Phys. Lett. B 756, 332 (2016); A. J. G. Carvalho, A. F. Ferrari, A. M. de Lima, J. R. Nascimento, and A. Yu. Petrov, Nucl. Phys. B942, 393 (2019).

[53] V. E. Mouchrek-Santos and M. M. Ferreira, Jr, Phys. Rev. D 95, 071701(R) (2017); J. Phys. Conf. Ser. 952, 012019 (2018).

[54] M. A. Anacleto, F. A. Brito, E. Maciel, A. Mohammadi, E. Passos, W. O. Santos, and J. R. L. Santos, Phys. Lett. B 785, 191 (2018).

[55] E. Passos, M. A. Anacleto, F. A. Brito, O. Holanda, G. B. Souza, and C. A. D. Zarro, Phys. Lett. B 772, 870 (2017).

[56] M. Haghighat, I. Motie, and Z. Rezaei, Int. J. Mod. Phys. A 28, 1350115 (2013).

[57] P. A. Bolokhov, M. Pospelov, and M. Romalis, Phys. Rev. D 78, 057702 (2008).

[58] R. Casana, M. M. Ferreira, Jr., E. Passos, F. E. P. dos Santos, and E. O. Silva, Phys. Rev. D 87, 047701 (2013).

[59] J. B. Araujo, R. Casana, and M. M. Ferreira, Jr., Phys. Rev. D 92, 025049 (2015).

[60] J. B. Araujo, R. Casana, and M. M. Ferreira, Jr, Phys. Lett. B 760, 302 (2016).

[61] J. B. Araujo, R. Casana, and M. M. Ferreira, Jr, Phys. Rev. D 97, 055032 (2018).

[62] Y. V. Stadnik, B. M. Roberts, and V. V. Flambaum, Phys. Rev. D 90, 045035 (2014).

[63] A. H. Gomes, V. A. Kostelecky, and A. J. Vargas, Phys. Rev. D 90, 076009 (2014).

[64] S. Aghababaei, M. Haghighat, and I. Motie, Phys. Rev. D 96, 115028 (2017).

[65] V. Alan Kostelecky and Z. Li, Phys. Rev. D 99, 056016 (2019).

[66] R. M. Sternheimer, Phys. Rev. 127, 1220 (1962).

[67] I. B. Khriplovich, Parity Nonconservation in Atomic Phenomena, 1st ed. (CRC Press, Philadelphia, 1991).

[68] R. Bluhm, V. Alan Kostelecky, C. D. Lane, and N. Russel, Phys. Rev. Lett. 88, 090801 (2002); Phys. Rev. D 68, 125008 (2003); V. A. Kostelecky and M. Mewes, Phys. Rev. D 66, 056005 (2002). 